\title{
Risk factors of poor cartilage regeneration in patients who underwent high tibial osteotomy combined with microfracture
}

\section{Ik-Sun Choi}

Chonnam National University Hwasun Hospital

\section{Pramod Shaligram Ingle}

Department of Orthopedic surgery, Manorama Hospital, ranaghat, Nadia, West Bengal India, India. Jong Keun Seon ( $\square$ seonbell@jnu.ac.kr)

Chonnam National University Hwasun Hospital https://orcid.org/0000-0002-7695-6098

\section{Eun-Kyoo Song}

Chonnam National University Hwasun Hospital

\section{Kyu-Jin Cho}

Chonnam National University Hwasun Hospital

\section{Seung-Min $\mathrm{Na}$}

Chonnam National University Hwasun Hospital

\section{Research article}

Keywords: High tibial osteotomy, Microfracture, Second-look arthroscopy, Cartilage regeneration, risk factor

Posted Date: November 26th, 2019

DOI: https://doi.org/10.21203/rs.2.10687/v2

License: (1) (1) This work is licensed under a Creative Commons Attribution 4.0 International License. Read Full License 


\section{Abstract}

Background: One of the option for treating unicompartmental osteoarthritis (OA) is high tibial osteotomy (HTO). HTO which can shift the mechanical axis to the lateral compartment, can performed with microfracture which provides cells capable of producing cartilage. And this procedure is one of the common combinations that orthopaedic surgeons are in practice recently. The purpose of this study was to evaluate the degree of regeneration of cartilage after performing microfracture (MF) with high tibial osteotomy (HTO) after 2-year follow up and identify the factors influencing the regeneration of cartilage after the procedure. We also evaluated whether the regenerated cartilage status affects clinical outcomes. Methods: A total of 81 cases that underwent second-look arthroscopy at the time of plate removal after MF and HTO with a minimum two-year follow-up were included. The patients were divided into two groups according to femoral cartilage regeneration. Multivariable logistic regression analyses were performend to identify independent factors that influence cartilage regeneration. We also compared differences in functional outcomes between the two groups. Results: On the femoral side, grade I cartilage regeneration was found in seven (8.6\%), grade II in 19 (23.5\%), grade III in 26 (32.1\%), and grade IV in 29 cases (35.8\%), resulting in $26(32.1 \%)$ patients belonging to well regenerated group (group A) and $55(67.9 \%)$ to the poorly regenerated group (group B). Among factors, the size of the cartilage lesion $(P=.011)$ and the presence of kissing lesions $(P=.027)$ significantly affected cartilage regeneration. There were no statistically significant differences between group A and group B in terms of KSS and WOMAC scores. Conclusions: A large cartilage defect and the presence of kissing lesions are associated with poor cartilage regeneration after combined MF and HTO surgery. However, the quality of regenerated cartilage does not affect functional outcomes in patients with MF and HTO. Keywords: High tibial osteotomy, Microfracture, Second-look arthroscopy, Cartilage regeneration, risk factor

\section{Background}

One of the option for treating unicompartmental osteoarthritis (OA) is high tibial osteotomy (HTO)[1]. As the literature has shown comparable results for both UKA and HTO[2,3], the latter has its own advantages in preserving native joints. Because of its simplicity, medial opening wedge HTO (OWHTO) has been increasingly used in recent years[4,5].

Cartilage damage of the knee joint due to $\mathrm{OA}$, is a challenging task for orthopaedic surgeons. While performing $\mathrm{HTO}$, concurrent arthroscopic procedures like shaving, microfracture, drilling, and abrasion chondroplasty are introduced[6]. The microfracture is simple and the most common way to bring bone marrow cells to the articular surface for the regeneration of cartilage[6]. High tibial osteotomy which can shift the mechanical axis to the lateral compartment, can performed with microfracture which provides cells capable of producing cartilage. This procedure is one of the common combinations that orthopaedic surgeons are in practice recently. Meanwhile, there are a few studies that have examined the nature of regenerated cartilage after microfracture together with $\mathrm{HTO}[7,8,9]$. Their results are conflicting with regard to the success of this combination in cartilage regeneration and functional outcomes. 
In this study, we evaluted the status of regenerated cartilage based on second-look arthroscopic findings in patients with medial OA of the knee, treated with combination of arthroscopic microfractures (MF) and HTO. The purpose of this study was to evaluate the degree of regeneration of cartilage after performing microfracture (MF) with high tibial osteotomy (HTO) after 2-year follow up and identify the factors influencing the regeneration of cartilage after the procedure. We also analyzed whether regenerated cartilage affects clinical outcomes. We hypothesized that the regeneration of cartilage is affected by several factors and that regenerated cartilage affects clinical outcomes.

\section{Methods}

This retrospective study was approved by institutional review board prior to commencement of the study. Total 182 patients underwent medial open wedge HTO and microfracture from 2007 to 2015 for medial unicompartmental OA (grade III and IV by the International Cartilage Research Society (ICRS) grading system during index arthroscopic finding) with varus deformity more than 5 degrees of Hip-Knee-Ankle (HKA) angle. Microfracture was done in both femoral and tibial side with ICRS grade III and IV. Among them, 137 patients underwent the plate removal and second-look arthroscopy. We excluded the patients with post-traumatic arthritis, combined ligament surgery or combined femoral osteotomy, or plate removal and patients who were followed-up less than 2 years, which left 81 patients. At the time of second-look arthroscopy, we evaluated the status of cartilage regeneration and reviewed patient records to analyze the factors affecting cartilage regeneration. All patients were operated at a single institute by two senior orthopaedic surgeons specialized in knee. A flowchart displaying the inclusion of patients in the study is shown in Figure 1.

\section{Surgical procedure and rehabilitation}

The subjects were operated in the supine position. Before performing HTO, a diagnostic arthroscopy was performed in all patients. Associated unrepairable meniscal tear was treated with partial or subtotal meniscectomy until the peripheral rim was stabilized. The cartilage defect was noted and categorized by ICRS grading. After arthroscopic shaving, the cartilage defect was debrided by using a small ring curette until an intact homogeneous peripheral rim was obtained. The central defect was then microfractured by a perforating owl with a 3 to $4 \mathrm{~mm}$ distance between the holes. After releasing the tourniquet pressure, we confirmed active bleeding from the microfracture holes before completing the surgery. Through anteromedial incision, a biplanar medial opening wedge osteotomy was carried out using a saw blade directed to the tip of the fibular head. Under fluoroscopic guidance, the opening of the deformity correction was targeted to achieve three to five degrees of a mechanical valgus postoperatively. The osteotomy was fixed with a MediFix locking plate (Medyssey., Jechon, Korea). The postoperative physiotherapy protocol consisted of a range of motion and muscle-strengthening exercises from the first postoperative day on, without weight-bearing mobilization, accompanied by the help of crutches from the second day, partial weight-bearing mobilization after 4 weeks, and full weight-bearing mobilization after 8 weeks. A knee brace was used until full weight bearing was initiated. 
When the osteotomy was healed completely, at about two years after index surgery, the plate was removed and second-look arthroscopy performed at the same time to examine the regenerated cartilage's characteristics. The cartilage regeneration was then classified according to the Cartilage Repair Assessment of ICRS Cartilage Injury Evaluation Package by the same orthopaedic surgeon who performed the previous osteotomy surgery. To investigate influential factors on cartilage regeneration, the patients were divided into two groups according to their cartilage status on second-look arthroscopy: group A (Well regenerated: grades I or II, Figure 2) and group B (Poorly regenerated: grades III or IV, Figure 3). The patients, radiological and articular factors affecting cartilage regeneration were then assessed in those two groups. Functional assessment was carried out using Knee Society Score (KSS) and the Western Ontario and McMaster Universities Osteoarthritis Index score (WOMAC), recorded preoperatively and at the last follow-up to compare the group A and group B.

\section{Assessment of factors}

We retrospectively assessed patient factors, including age, sex, body mass index (BMI), radiologic factors (pre- and postoperative HKA angle, correction angle, pre- and postoperative tibial slope), and articular factors (meniscectomy, root tear of meniscus, size of cartilage lesion, presence of kissing lesions) with regard to their association with cartilage regeneration.

The radiological factors were measured digitally on PACS (Picture Archiving and Communication System; INFINITT Healthcare, Seoul, Korea). Preoperative and postoperative HKA angles were measured as the angle between the mechanical axis of the femur and the tibia with negative values for varus and positive values for valgus. The tibial slope angle was calculated as described by Brandon et al.[10] on a standard lateral knee radiograph as the angle subtended between the tibial anatomical long axis, in the line between the center of the anteroposterior diameter measured just below the tibial tuberosity and the center of the anteroposterior diameter measured $5 \mathrm{~cm}$ distal to the former, and a line drawn from the medial tibial plateau. The number of patients who underwent either partial or subtotal meniscectomy was recorded. The size of the cartilage lesion (defect $\geq 2.5 \mathrm{~cm} 2$ or less), and the presence of a kissing lesion were noted on intraoperative arthroscopic findings at the time of index surgery.

\section{Statistical analysis}

Statistical analysis was carried out using SPSS Statistics for Windows (version 22; IBM, Armonk, NY). A univariate and multivariate logistic regression model was employed to analyze the factors affecting cartilage regeneration. Postoperative functional improvement was assessed using paired sample $t$ tests. To compare functional outcomes between the two groups, an independent sample t-test was calculated. A $P$ value $<.05$ was considered statistically significant.

\section{Results}

The demographic data are shown in Table 1. There were 73 patients (22 male and 51 females) with eight bilateral cases for a total of 81 cases. 
Table 1. Characteristics of Patients

Characteristic

Value

Number of Cases

81

Male 25

Female 56

Age (yr), mean \pm SD

$55.7 \pm 5.2$

Body mass index $\left(\mathrm{kg} / \mathrm{m}^{2}\right)$, mean $\pm \mathrm{SD}$

$25.8 \pm 3.3$

Time to plate removal and second-look arthroscopy (mo), mean \pm $25.6 \pm 5.5$ SD

Follow-up (mo), mean \pm SD

$38.5 \pm$ 20.1

Preoperative HKA angle (degrees), mean \pm SD $-6.0 \pm 2.1$

Postoperative HKA angle (degrees), mean \pm SD $3.5 \pm 1.0$

Correction angle (degrees), mean \pm SD $9.4 \pm 1.9$

Preoperative tibial slope (degrees), mean \pm SD $7.0 \pm 2.2$

Postoperative tibial slope (degrees), mean \pm SD $7.7 \pm 2.2$

The values are expressed as the mean \pm standard deviation, unless stated otherwise. Negative values indicate varus angulation

SD: standard deviation, HKA angle: Hip-Knee-Ankle angle

The distribution of cartilage lesions on femoral and tibial articular surfaces as per ICRS classification at the time of index surgery is shown in Table 2. The status of cartilage regeneration on second-look arthroscopy according to the Cartilage Repair Assessment of ICRS evaluation package system is shown in Table 3. On the femoral side, grade I cartilage regeneration was found in 7 cases (8.6\%), grade II in 19 cases $(23.5 \%)$, grade III in 26 cases (32.1\%), and grade IV in 29 cases (35.8\%), resulting in 26 cases (32.1\%) belonging to group $A$ and 55 subjects (67.9\%) to group $B$. 
Table 2. Frequency of ICRS Grades in Articular Cartilage at the Time of Diagnostic Arthroscopy $\left(1^{\text {st }}\right)$

\begin{tabular}{llllll}
\hline ICRS Grades at the Time of Diagnostic Arthroscopy \\
\hline \multicolumn{7}{c}{ Grade IGrade IIGrade IIIGrade IVTotal } \\
\hline Femoral side0 & 0 & 32 & 49 & 81 \\
Tibial side & 45 & 19 & 6 & 11 & 81 \\
& & & & \\
\hline
\end{tabular}

The values are the number of cases.

ICRS: International Cartilage Repair Society

Table 3. Cartilage Repair Assessment after Second-Look Arthroscopy (2 $\left.{ }^{\text {nd }}\right)$

\begin{tabular}{lccccc}
\hline Cartilage Repair Assessment \\
\hline \multicolumn{7}{c}{ Grade IGrade IIGrade IIIGrade IVTotal } \\
\hline Femoral side7 & 19 & 26 & 29 & 81 \\
Tibial side & 47 & 20 & 6 & 8 & 81 \\
\hline
\end{tabular}

The values are the number of cases.

On the tibial side, grade I cartilage regeneration was present in 47 cases $(58.0 \%)$, grade II in 20 cases (24.7\%), grade III in 6 cases (7.4\%), and grade IV in 8 cases (9.9\%) which led to group A of 67 cases $(82.7 \%)$ and 14 cases $(17.3 \%)$ in group B. Meniscectomy was performed 11 cases in group A and 26 cases in group B. Root tear of meniscus was accompanied 3 cases in group A and 19 cases in group B.

\section{Assessment of factors}

In terms of influential factors (patients, radiological and articular factors) for cartilage regeneration on the femoral side, univariate logistic regression analysis is shown in Table 4. and multivariate logistic regression analysis is shown in Table 5.

Table 4. Univariate Logistic Regression of Factors Affecting Cartilage Regeneration 


\begin{tabular}{lccc}
\hline Factors & \multicolumn{2}{l}{ Odds ratio95 \% confidence intervalPvalue } \\
\hline Age & 0.672 & $0.794-1.095$ & .726 \\
Gender & 0.825 & $0.019-0.850$ & .062 \\
Body mass index & 1.573 & $1.151-2.150$ & .004 \\
\hline Preoperative HKA angle & 0.611 & $0.055-6.786$ & .384 \\
\hline Postoperative HKA angle & 0.305 & $0.019-3.452$ & .120 \\
\hline Correction angle & 1.677 & $0.149-18.904$ & .343 \\
\hline Preoperative tibial slope & 0.976 & $0.059-3.849$ & .115 \\
\hline Postoperative tibial slope & 1.719 & $0.230-0.825$ & .027 \\
\hline Meniscectomy & 0.304 & $0.056-1.644$ & .187 \\
\hline Root tear of meniscus & 0.381 & $0.035-4.107$ & .511 \\
\hline Size of cartilage defect $\geq 2.5 \mathrm{~cm}^{2} 5.024$ & $0.442-57.099$ & .048 \\
\hline Kissing lesions & 0.009 & $0.017-0.110$ & .000 \\
\hline
\end{tabular}

Table 5. Multivariate Logistic Regression of Factors Affecting Cartilage Regeneration Factors Odds ratio95 \% confidence interval $P$ value

\begin{tabular}{lccc}
\hline Body mass index & 1.455 & $0.674-3.138$ & .339 \\
\hline Preoperative tibial slope & 0.976 & $0.452-2.104$ & .851 \\
\hline Postoperative tibial slope & 1.719 & $0.393-7.516$ & .472 \\
Size of cartilage defect $\geq 2.5 \mathrm{~cm}^{2} 3.071$ & $1.291-7.306$ & .011 \\
Kissing lesions & 2.483 & $1.107-5.569$ & .027 \\
\hline
\end{tabular}

The two most important factors affecting cartilage regeneration were the size of cartilage lesions $(\geq 2.5$ $\mathrm{cm} 2)(P=.011)$ and the presence of a kissing lesion $(P=.027)$. 
The mean preoperative KSS and WOMAC scores were $67.5 \pm 11.2$ and $33.1 \pm 15.4$, respectively. At the most current follow-up, KSS and WOMAC scores improved to $87.4 \pm 9.2$ and $11.4 \pm 7.7$, respectively. The pre- and postoperative values' difference was significant at the last follow-up $(P<.001)$. There was no statistically significant difference between the groups $A$ and $B$ in terms of postoperative functional scores (KSS, $P=.65$; WOMAC, $P=.89$ ).

\section{Discussion}

The most important finding of our study is that although Cartilage Repair Assessment grade III or IV cartilage regeneration could be found in about one third of all cases, there were no functional benefits compared to poor cartilage regeneration. The main focus of this research was to determine the factors associated with cartilage regeneration and we found that cartilage lesions $\geq 2.5 \mathrm{~cm} 2$ and the presence of a kissing lesion were strongly associated with poor cartilage regeneration, compared with age, sex, BMI, pre- and postoperative HKA angle, correction angle, pre- and postoperative tibial slope, meniscectomy, and root tear of the meniscus. Although there are a few studies to examine factors associated with cartilage regeneration after HTO, microfracture under arthroscopy was not performed. Recently, Kumagai et al. reported that the factors that affected cartilage regeneration after $\mathrm{HTO}$, were such as postoperative limb alignment, preoperative cartilage degeneration grade, and BMI, but not by age or gender[11]. Kim et al. found that regeneration of articular cartilage was associated with a smaller mean preoperative varus mechanical tibiofemoral angle and lower BMI for the medial femoral condyle[12]. Bae et al. performed a study of microfracture in an OA knee with a minimum 10-year follow-up and reported that cartilage lesions $>2 \mathrm{~cm} 2$ and a preoperative mechanical axis which bisected the total width of the tibia more than $25 \%$ were associated with poor results. But BMI and partial meniscectomy was not contributing factors[13].

According to the patient factors, consistent with Bae et al.[13], we could not find any effect of BMI on cartilage regeneration, contrary to Kumagai et al.[11] and Kim et al.[12]. The relatively low BMI of our study sample might have been the reason why there was no association with cartilage regeneration. In a study of arthroscopic abrasion arthroplasty combined with HTO, Akizuki et al. showed that age was the only factor influencing tibial cartilage[14]. Though some studies have demonstrated a better cartilage regeneration in younger individuals, especially $<35$ years, our investigation revealed no age effect since we had included patients with high-grade OA, all of them above 40 years of age[6].

A large varus deformity as determined by the preoperative HKA angle is also one of the contributing factors for the regeneration of the cartilage in most studies. Kumagai et al. and Koshino et al. have also shown that mature regeneration was observed more frequently in the knees with more than $1^{\circ}$ of mechanical valgus angulation after osteotomy than in those with less than $1^{\circ}[15]$. However, Tsukada and Wakui recently reported no significant differences in the ratio of cartilage repair between overcorrected (defined as knees with mechanical valgus angulation $\geq 8^{\circ}$ ) and moderately corrected (defined as knees with mechanical valgus angulation $\leq 8^{\circ}$ ) knees[16]. Our study did not show any association between clinical outcome and cartilage regeneration with pre- and postoperative HKA angles as well as the 
correction angle. The goal of our corrective osteotomy was to achieve three to five degrees valgus angulation and hence most of our patients had overcorrected knees. It is well known that an open wedge HTO tends to increase, and a closed wedge HTO tends to decrease the postoperative slope[17,18]. Although the tibial slope increased after surgery, neither the preoperative tibial slope nor its change affected cartilage regeneration postoperatively.

Meniscal root tear is commonly associated with high-grade degenerative arthritis. Menisci are important for resisting axial load by generating hoop stresses. Meniscal root tear alters the knee's biomechanics[19,20,21,22]. A root tear of the meniscus is almost equivalent to total meniscectomy, increasing tibiofemoral contact pressure as demonstrated by Allaire et al.[19]. Takahashi et al. showed that tears in the posterior root of the medial meniscus were independent risk factors for cartilage degeneration as shown on T1p MRI scans[23]. Though statistically not significant, most cases with poor cartilage regeneration in our study had a root tear of the medial meniscus. Krych et al. reported that nonoperative management of the medial meniscus root tear is associated with worsening arthritis and poor functional outcomes at a five-year follow-up[24]. Most of the root tears in our study were managed with either partial or subtotal meniscectomy to obtain a stable peripheral rim. Sterett et al. reported that patients with a tear of the medial meniscus at the time of chondral resurfacing were 9.2 times more likely to undergo TKA than those without tear[25]. But similar to Bae et al.[13], our study also showed that meniscectomy in general does not affect cartilage regeneration.

Among all factors, the most significant were the size of the cartilage lesion and the presence of a kissing lesion. In our study, a cartilage defect $\geq 2.5 \mathrm{~cm} 2$ was the most consistent factor associated with poor cartilage regeneration. Moreover, when the kissing lesions is present, it can possibly affect cartilage regeneration by disturbing the stable position of marrow stem cells during the regeneration process. Like in our study, most investigations assessing cartilage regeneration have shown that a larger cartilage defect is associated with poor cartilage regeneration[11,13,25].

Similar to Schuster et al.[26], our study revealed that there were no statistically significant differences in functional outcomes between well regenerated and poorly regenerated groups. This can be interpreted that postoperative functional improvement is mainly due to the medial compartment's unloading after HTO.

\section{Limitations}

This study had several limitations. First, it was a retrospective study with limited cases. Second, tibial cartilage regeneration was not taken into account. Third, cartilage factors were not treated in detail. Fourth, we did not compare with the control group which patients who had undergone HTO without MF.

\section{Conclusions}

A large cartilage defect $(\geq 2.5 \mathrm{~cm} 2)$ and the presence of a kissing lesion are associated with poor cartilage regeneration after combined HTO and MF surgery. The quality of regenerated cartilage does not 
affect functional outcomes. Therefore, surgeons should determine the necessity of microfracture based on risk factors, especially with respect to the size of the cartilage defect and the presence of a kissing lesion at the time of HTO.

\section{Abbreviations}

OA: Osteoarthritis

HTO: High tibial osteotomy

MF: Mirofracture

OWHTO: Opening wedge High tibial osteotomy

ICRS: International Cartilage Research Society

HKA: Hip-Knee-Ankle

KSS: Knee Society Score

WOMAC: Western Ontario and McMaster Universities Osteoarthritis Index

BMl: Body mass index

\section{Declarations}

\section{Ethics approval and consent to participate}

The study was conducted with the approval of the institutional review board(IRB) of Chonnam National University Hwasun Hospital. All participants signed written consent to participate in the study. All rights of the patients were protected against any kind of disadvantage and individual matters.

\section{Consent for publication}

Not applicable.

\section{Availability of data and material}

The datasets used and/or analyzed during the current study are available from the corresponding author on reasonable request.

\section{Competing interests}

The authors declare that they have no competing interests.

\section{Funding}


There was no funding associated with this study.

\section{Author's contributions}

1. ISC: Analysis of data and writing manuscript and review.

2. PSI: Analysis of data and writing manuscript

3. EKS: Make concepts and design of study

4. JKS: Make concepts and design of study. Review and correction of draft manuscript

5. KJC: Data collection and analysis

6. SMN: Analysis of data and writing manuscript

\section{Acknowledgements}

The authors thanks all clinical researchers involved in the research we included in this article. This study was not supported by any company or grant.

\section{References}

1. Kfuri M, Lobenhoffer P. High tibial osteotomy for the correction of varus knee deformity. J Knee Surg 2017;30:409-20.

2. Santoso MB, Wu L. Unicompartmental knee arthroplasty, is it superior to high tibial osteotomy in treating unicompartmental osteoarthritis? A meta analysis and systemic review. J Orthop Surg Res 2017;12:552-9.

3. Rodriguez-Merchan EC. Unicompartmental Knee Osteoarthritis (UKOA): Unicompartmental Knee Arthroplasty (UKA) or High Tibial Osteotomy (HTO)? Arch Bone Jt Surg 2016;4:307-13.

4. Sun H, Zhou L, Li F, Duan J. Comparison between closing-wedge and opening-wedge high tibial osteotomy in patients with medial knee osteoarthritis: a systematic review and meta- analysis. $J$ Knee Surg 2017;30:158-65.

5. Agarwala S, Sobti A, Naik S, Chaudhari S. Comparison of closing-wedge and opening-wedge high tibial osteotomies for medial compartment osteoarthritis of knee in Asian population: Mid- term follow-up. J Clin Orthop Trauma 2016;7:272-5.

6. Bekkers JE, Inklaar M, Saris DB. Treatment selection in articular cartilage lesions of the knee: a systematic review. Am J Sports Med 2009;37:S148-55.

7. Jung WH, Takeuchi R, Chun CW, Lee JS, Jeong JH. Comparison of results of medial opening- wedge high tibial osteotomy with and without subchondral drilling. Arthroscopy 2015;31:673-9.

8. Spahn G, Klinger HM, Harth P, Hofmann Cartilage regeneration after high tibial osteotomy. Results of an arthroscopic study. Z Orthop Unfall 2012;150:272-9.

9. Matsunaga D, Akizuki S, Takizawa T, Yamazaki I, Kuraishi J. Repair of articular cartilage and clinical outcome after osteotomy with microfracture or abrasion arthroplasty for medial gonarthr Knee 
2007; 14:465-71.

10. Brandon L, Haynes T, Bonamo R, Flynn I, Barrett R, Sherman F. The Association Between PosteriorInferior Tibial Slope and Anterior Cruciate Ligament Insufficiency. Arthroscopy 2006;22(8):894-9

11. Kumagai K, Akamatsu Y, Kobayashi H, Kusayama Y, Koshino T, Saito T. Factors affecting cartilage repair after medial opening-wedge high tibial osteotomy. Knee Surg Sports Traumatol Arthrosc 2017;25:779-

12. $1 \mathrm{Kim}$ KI, Seo MC, Song SJ, Bae DK, Kim DH, Lee S Change of chondral lesions and predictive factors after medial open-wedge high tibial osteotomy with a locked plate system. Am J Sports Med 2017;45:1615-21.

13. Bae DK, Song SJ, Yoon KH, Heo DB, Kim TJ. Survival analysis of microfracture in the osteoarthritic knee-minimum 10-year follow- Arthroscopy 2013;29:244-50.

14. Akizuki S, Yasukawa Y, Takizawa T. Does arthroscopic abrasion arthroplasty promote cartilage regeneration in osteoarthritic knees with eburnation? A prospective study of high tibial osteotomy with abrasion arthroplasty versus high tibial osteotomy alone. Arthroscopy 1997;13;9-17.

15. Koshino T, Wada S, Ara Y, Saito T. Regeneration of degenerated articular cartilage after high tibial valgus osteotomy for medial compartmental osteoarthritis of the knee. Knee 2003;10:229-36.

16. Tsukada S, Wakui M. Is overcorrection preferable for repair of degenerated articular cartilage after open-wedge high tibial osteotomy? Knee Surg Sports Traumatol Arthrosc 2017;25:785-92.

17. LaPrade RF, Oro FB, Ziegler CG, Wijdicks CA, Walsh MP. Patellar height and tibial slope after openingwedge proximal tibial osteotomy: a prospective study. Am J Sports Med 2010;38:160-

18. Marti CB, Gautier E, Wachtl SW, Jakob RP. Accuracy of frontal and sagittal plane correction in openwedge high tibial osteotomy. Arthroscopy 2004;20:366-72.

19. Allaire R, Muriuki M, Gilbertson L, Harner CD. Biomechanical consequences of a tear of the posterior root of the medial meniscus. Similar to total meniscectomy. J Bone Joint Surg Am 2008;90:1922-31.

20. Forkel P, Herbort M, Schulze M, Rosenbaum D, Kirstein L, Raschke M, Petersen W. Biomechanical consequences of a posterior root tear of the lateral meniscus: stabilizing effect of the meniscofemoral ligament. Arch Orthop Trauma Surg 2013;133:621-6.

21. Harner CD, Mauro CS, Lesniak BP, Romanowski JR. Biomechanical consequences of a tear of the posterior root of the medial meniscus. Surgical technique. J Bone Joint Surg Am 2009;91:257-70.

22. Ding C, Martel-Pelletier J, Pelletier JP, Abram F, Raynauld JP, Cicuttini F, Jones G. Knee meniscal extrusion in a largely non-osteoarthritic cohort: association with greater loss of cartilage volume. Arthritis Res Ther 2007;9:R21

23. 23. Takahashi K, Hashimoto S, Nakamura H, Mori A, Sato A, Majima T, Takai S. Medial meniscal posterior root/horn radial tears correlate with cartilage degeneration detected by $\mathrm{T} 1 \rho$ relaxation mapping. Eur J Radiol 2015;84:1098-

24. Krych AJ, Reardon PJ, Johnson NR, Mohan R, Peter L, Levy BA, Stuart MJ. Non-operative management of medial meniscus posterior horn root tears is associated with worsening arthritis and 
poor clinical outcome at 5-year follow- Knee Surg Sports Traumatol Arthrosc 2017;25:383-9.

25. Sterett WI, Steadman JR, Huang MJ, Matheny LM, Briggs Chondral resurfacing and high tibial osteotomy in the varus knee: survivorship analysis. Am J Sports Med 2010;38:1420-4.

26. Schuster P, Schulz M, Mayer P, Schlumberger M, Immendoerfer M, Richter J. Open-wedge high tibial osteotomy and combined abrasion/microfracture in severe medial osteoarthritis and varus malalignment: 5-year results and arthroscopic findings after 2 year Arthroscopy 2015;31:1279-88. 